\title{
Changes in antiepileptic drug prescriptions over a decade in childbearing women in Lombardy Region, Italy
}

\author{
Antonio Clavenna ${ }^{1}$, Rita Campi ${ }^{1}$, Daria Putignano ${ }^{1}$, Ida Fortino ${ }^{2}$, and Maurizio Bonati ${ }^{1}$ \\ ${ }^{1}$ Istituto di Ricerche Farmacologiche Mario Negri \\ ${ }^{2}$ Lombardy Region
}

July 3, 2021

\begin{abstract}
Aim To describe the antiepileptic drug (AED) prescription pattern in pregnant women and women of childbearing age in the 2010-2019 period in the Lombardy region, Italy. Methods The Lombardy region administrative healthcare databases (20102019) were analysed. AEDs were classified as drugs belonging to the N03A subgroup of the Anatomical Therapeutic Chemical Classification System. Women 15-49 years old were considered as women of childbearing age, while exposure during pregnancy was estimated taking into account the 12 months before delivery (International Classification of Diseases, Ninth Revision, Clinical Modification, ICD-9-CM codes in the diagnosis record from 650 to 659). Results During 2019, 16,605 women of childbearing age (prevalence: 14.8least one AED prescription. Pregabalin was the most widely used antiepileptic in women of childbearing age $(22.3 \%)$, followed by valproic acid $(20.0 \%)$. The prevalence of AED prescription in pregnant women was 3.8 commonly prescribed drugs. The prevalence of AED prescription did not change from 2010 to 2019 in women of childbearing age or in pregnant women. Valproic acid was one of the most used AEDs in pregnancy until 2016, after which its prescription declined from $19 \%$ to $14 \%$ of AED users. Conclusions Despite the decrease in valproic acid prescription over time, this drug is still among the most used AEDs, in particular in women of childbearing age. Educational interventions for healthcare professionals and women are needed in order to reduce the risk of unplanned pregnancy.
\end{abstract}

\section{Hosted file}

Clavenna_Manuscript_def.docx available at https://authorea.com/users/423537/articles/528891changes-in-antiepileptic-drug-prescriptions-over-a-decade-in-childbearing-women-inlombardy-region-italy 


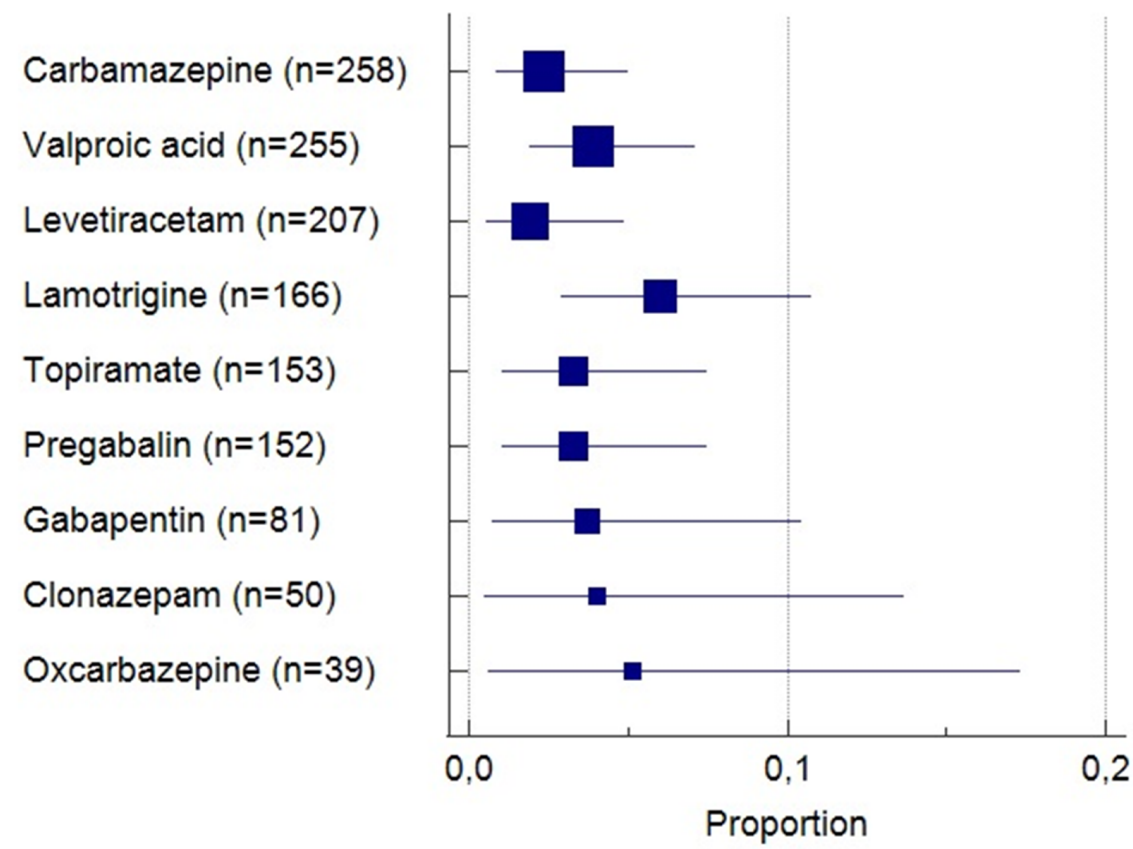

